\title{
Features of Correlation Analysis of Images and Video Sequences
}

\author{
Andrey V. Bogoslovsky, Andrey V. Ponomarev, \\ Irina V. Zhigulina and Vladimir A. Sukharev* \\ Military Education and Research Centre of Military-Air Forces \\ "Military-Air Academy \\ Named After Professor N.E. Zhukovsky and Yu.A. Gagarin" \\ 54a Starykh Bolshevikov Str., Voronezh, 394064, Russia
}

Received 22.06.2018, received in revised form 17.08.2018, accepted 12.10.2018

The paper deals with 2D-dimensional autocorrelation functions of $2 D$-dimensional video signals with allowance for the finiteness of the field of view, and it is established that the limits of integration need to be adjusted. It is shown that the autocorrelation function can be used to estimate the location of the object relative to the edges of the image. Possible variants of the object displacement along the image field are considered, the autocorrelation function is determined through the change in the area of the object when it leaves the field of view. Variable substitutions were introduced to find the difference between the autocorrelation functions in the processing of video sequences. Stable areas are identified that change only their location depending on the parameters of the object's movement and its position relative to the center of the frame. The results are consistent for continuous and discrete models. The stability of the proposed method for identifying the motion on the difference in the autocorrelation functions to the change in the shape of the object is shown.

Keywords: image, videosequence, image energycharacteristics, signalspatiallimit, 2D-autocorrelation function.

Citation: Bogoslovsky A.V., Ponomarev A.V., Zhigulina I.V., Sukharev V.A. Features of correlation analysis of images and video sequences, J. Sib. Fed. Univ. Eng. technol., 2018, 11(7), 811-822. DOI: 10.17516/1999-494X-0096.

(C) Siberian Federal University. All rights reserved

This work is licensed under a Creative Commons Attribution-NonCommercial 4.0 International License (CC BY-NC 4.0).

* Corresponding author E-mail address: p-digim@mail.ru 


\title{
Особенности корреляционного анализа изображений
}

\section{и видеопоследовательностей}

\author{
А.В. Богословский, А.В. Пономарев, \\ И.В. Жигулина, В.А. Сухарев \\ Военный учебно-научный центр Военно-воздушных сил \\ «Военно-воздушная академия \\ имени профессора Н.Е. Жуковского и Ю.А. Гагарина» \\ Россия, 394064, Воронеж, ул. Старых Большевиков, 54 а
}

\begin{abstract}
В работе рассмотрены двумерные автокорреляционные функииидвумерныхвидеосигналов с учетом финитности поля зрения и установлена необходимость корректировки пределов интегрирования. Показано, что по функичии автокорреляции можно оценить расположение объекта относительно краев изображения. Рассмотрены возможные варианты смещчения объекта по полю изображения, определена функция автокорреляциии через изменение площуади объекта при выходе его за предель поля изображения. Введены замены переменных для нахождения разности автокорреляциионных функичй при обработке видеопоследовательностей. Выделены стабильные области, которые меняют только свое расположение в зависимости от параметров движения объекта и его положения относительно иентра кадра. Проведено согласование результатов для непрерывных и дискретных моделей. Показана устойчивость предлагаемого метода идентификацчии движения по разности автокорреляциионных функичй к изменению формы объекта.

Ключевые слова: изображение, видеопоследовательность, энергетические характеристики изображения, пространственная ограниченность сигнала, двумерная функция автокорреляции.
\end{abstract}

\section{Введение}

Изображения по своей физической природе ограничены по пространственным координатам. Обычно этот факт не принимается во внимание, и считается, что края изображения находятся достаточно далеко, чтобы их не учитывать, либо их влияние сводится к различным краевым эффектам, приводящим к отрицательным артефактам.

Однако в исследованиях $[1,2]$ показано, что при обработке видеопоследовательностей ограниченность кадров является дополнительным преимуществом для обнаружения динамических объектов и определения их параметров. Решение такой задачи связано с нахождением разности автокорреляционных функций. Двумерные автокорреляционные функции могут быть использованы и при обработке 2D-изображений [3].

В работе [4] обоснована необходимость учета финитности поля зрения при нахождении автокорреляционных функций одномерных видеосигналов, для чего требуется корректировка пределов интегрирования. Но в двумерном случае появляется новое качество - форма объектов, отсутствующая при одномерном анализе. Кроме того, для дискретного изображения характерно наличие «переходных» пикселей, усложняющих процесс анализа изображения. У непрерывного изображения переходные пиксели отсутствуют, что позволяет находить автокорреляционные функции в общем виде. 
Целью статьи является анализ двумерных автокорреляционных функций изображений и видеопоследовательностей, а также согласование результатов для непрерывных и дискретных моделей.

\section{Постановка задачи}

На рис. 1 представлено изображение простейшего объекта - произвольного четырехугольника $A B C D$ яркостью $\beta$ - на равномерном фоне яркостью $\alpha$. Размер всего изображения $(2 M+1) \times(2 N+1)$; координаты вершин прямоугольника: $A\left(l_{x}, l_{y}\right), B\left(u_{x}, u_{y}\right), C\left(r_{x}, r_{y}\right), D\left(d_{x}, d_{y}\right)$.

В одномерном случае функция автокорреляции является четной функцией аргумента $k$. В двумерном случае она должна обладать, как и энергетический спектр, центральной симметрией, поэтому будем рассматривать автокорреляционную функцию $J\left(k_{1}, k_{2}\right)$ для $0 \leq k_{1} \leq 2 M$; $-2 N \leq k_{2} \leq 2 N$.

На рис. 2 схематично показано, как устанавливаются пределы интегрирования для двух случаев: $k_{2} \geq 0$ и $k_{2}<0$.

Найдем автокорреляционную функцию для каждого из указанных случаев:

$$
\begin{aligned}
& J\left(k_{1} ; k_{2} \geq 0\right)= \int_{-M+k_{1}}^{M} \int_{-N+k_{2}}^{N}\left\{\alpha+(\beta-\alpha) \cdot\left[\mathbf{1}\left(y-a_{1} x-b_{1}\right)-\mathbf{1}\left(y-a_{2} x-b_{2}\right)\right] \times\left[\mathbf{1}\left(y-c_{2} x-d_{2}\right)-\right.\right. \\
&\left.\left.-\mathbf{1}\left(y-c_{1} x-d_{1}\right)\right]\right\} \cdot\left\{\alpha+(\beta-\alpha) \cdot\left[\mathbf{1}\left(y-k_{2}-a_{1} x-b_{1}+a_{1} k_{1}\right)-\mathbf{1}\left(y-k_{2}-a_{2} x-b_{2}+a_{2} k_{1}\right)\right] \times \quad\right. \\
&\left.\times\left[\mathbf{1}\left(y-k_{2}-c_{2} x+c_{2} k_{1}-d_{2}\right)-\mathbf{1}\left(y-k_{2}-c_{1} x+c_{1} k_{1}-d_{1}\right)\right]\right\} d x d y ; \\
& J\left(k_{1} ; k_{2}<0\right)= \int_{-M+k_{1}}^{M} \int_{-N}^{N+k_{2}}\left\{\alpha+(\beta-\alpha) \cdot\left[\mathbf{1}\left(y-a_{1} x-b_{1}\right)-\mathbf{1}\left(y-a_{2} x-b_{2}\right)\right] \times\left[\mathbf{1}\left(y-c_{2} x-d_{2}\right)-\right.\right. \\
&\left.\left.-\mathbf{1}\left(y-c_{1} x-d_{1}\right)\right]\right\} \cdot\left\{\alpha+(\beta-\alpha) \cdot\left[\mathbf{1}\left(y-k_{2}-a_{1} x-b_{1}+a_{1} k_{1}\right)-\mathbf{1}\left(y-k_{2}-a_{2} x-b_{2}+a_{2} k_{1}\right)\right] \times \quad\right. \\
&\left.\times\left[\mathbf{1}\left(y-k_{2}-c_{2} x+c_{2} k_{1}-d_{2}\right)-\mathbf{1}\left(y-k_{2}-c_{1} x+c_{1} k_{1}-d_{1}\right)\right]\right\} d x d y, \quad \text { если } k_{2}<0,
\end{aligned}
$$

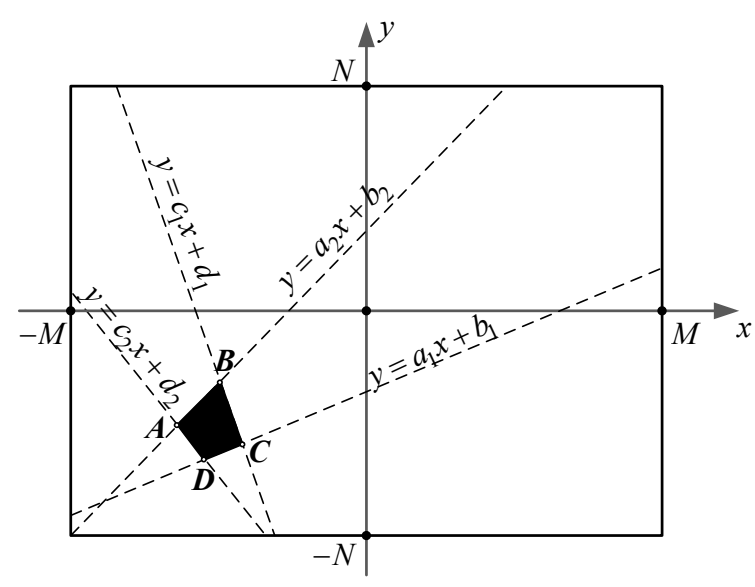

Рис. 1. Изображение объекта на равномерном фоне

Fig. 1. Image of the object on a uniform background 
где $\mathbf{1}(x, y)$ - двумерная функция Хевисайда, $\mathbf{1}(x, y)=\left\{\begin{array}{l}1 \text { при } x \geq 0, y \geq 0, \\ 0 \text { в противном случае. }\end{array}\right.$

При нахождении функции автокорреляции возможно несколько вариантов «смещения» объекта $A B C D$ (рис. 1) по полю изображения, они приведены в табл. 1 для $k_{1} \geq 0$.

Для всех случаев из табл. 1 характерны дополнительные ограничения на аргументы $k_{1}, k_{2}$. Так, для случаев 1-3 они описываются системами неравенств:

$$
\begin{array}{ll}
\text { случай 1: } & \left\{\begin{array}{l}
k_{2} \leq a_{2} k_{1}+N-M a_{2}-b_{2} ; \\
k_{2} \geq c_{2} k_{1}-N-M c_{2}-d_{2} ;
\end{array}\right. \\
\text { случай 2: } & \left\{\begin{array}{l}
k_{2} \geq N+c_{2} k_{1}-d_{2} ; \\
k_{2} \geq a_{1}\left(k_{1}-M\right)+N-b_{1} ;
\end{array}\right. \\
\text { случай 3: } & \left\{\begin{array}{l}
k_{2} \leq a_{2} k_{1}-b_{2}-N ; \\
k_{2} \leq c_{1}\left(k_{1}-M\right)-d_{1}-N .
\end{array}\right.
\end{array}
$$

Области, соответствующие ограничениям (3)-(5), выделены на рис. 3.

Если значения $k_{1}$ и $k_{2}$ таковы, что объект «выходит» за предель поля изображения, то величина корреляционного интеграла будет зависеть от изменения площади объекта.

На рис. 4 схематично показано «смещение» объекта, соответствующее случаю 1 из табл. 1.

Таким образом, при нахождении функций автокорреляции в непрерывном случае достаточно установить зависимость площади объекта от значений аргументов $k_{1}$ и $k_{2}$.

Определим изменение площади объекта при выходе его за пределы поля изображения.

Площадь объекта - произвольного четырехугольника $A B C D$ (рис. 1) - можно описать как функцию только от $k_{1}$ (случай 1 ) либо от $k_{1}$ (случаи 2,3 ). При изменении $k_{1}$ или $k_{2}$ в силу особенностей рассматриваемого объекта его можно разбить на три области $J_{1}, J_{2}, J_{3}$, последовательно «покидающие» поле изображения, что схематично представлено на рис. 5.

Площади областей $J_{1}, J_{2}, J_{3}$, находящихся за пределами поля изображения, определяются следующим образом:
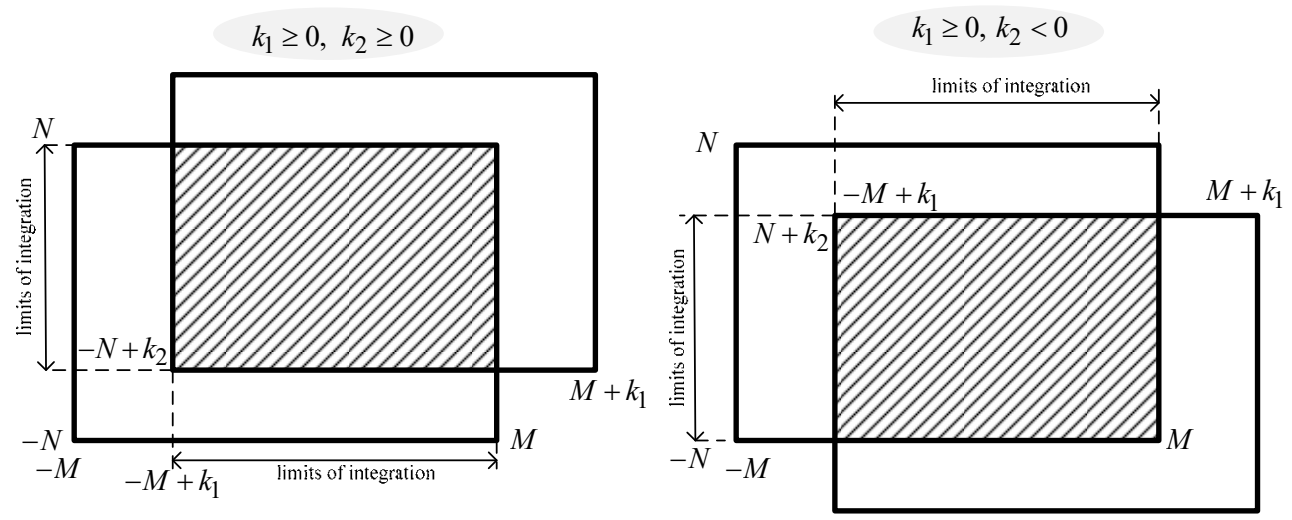

Рис. 2. Определение пределов интегрирования

Fig. 2. Determination of integration limits 


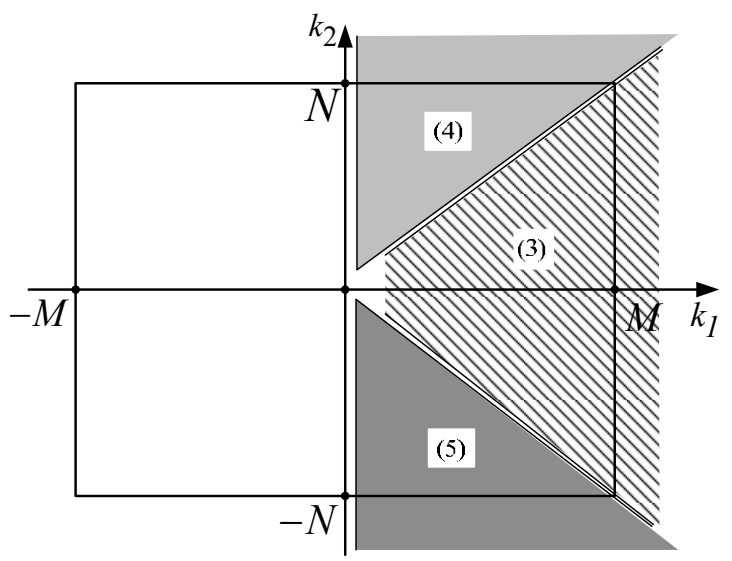

Рис. 3. Области изменений аргументов $k_{1}$ и $k_{2}$

Fig. 3. Areas of argument changes $k_{1}$ and $k_{2}$

Таблица 1. Варианты смещения объекта по полю изображения

Table 1. Variants of object displacement on an image field

\begin{tabular}{|c|c|c|c|}
\hline $\begin{array}{c}\text { № } \\
\Pi / \Pi\end{array}$ & \multicolumn{2}{|r|}{ Направление смещения объекта } & Условия для аргумента $k_{2}$ \\
\hline 1 & \multicolumn{2}{|c|}{ объект «перемещается» только через линию $x=M$} & $k_{2}<0$ либо $k_{2} \geq 0$ \\
\hline 2 & \multicolumn{2}{|c|}{ объект «перемещается» только через линию $y=N$} & $k_{2}>0$ \\
\hline 3 & \multicolumn{2}{|c|}{ объект «перемещается» только через линию $y=-N$} & $k_{2}<0$ \\
\hline 4 & \multirow{2}{*}{$\begin{array}{l}\text { перемещение } \\
\text { через углы } \\
\text { изображения }\end{array}$} & $\begin{array}{l}\text { объект «перемещается» через верхний } \\
\text { правый угол, т.е. одновременно через } \\
\text { линии } x=M \text { и } y=N\end{array}$ & $k_{2}>0$ \\
\hline 5 & & $\begin{array}{l}\text { объект «перемещается» через нижний } \\
\text { правый угол, т.е. одновременно через } \\
\text { линии } x=M \text { и } y=-N\end{array}$ & $k_{2}<0$ \\
\hline
\end{tabular}

$$
\begin{aligned}
& \left\{\begin{array}{l}
J_{1}\left(k_{1}\right)=0,5\left[\left(M-k_{1}\right)\left(c_{1}-a_{1}\right)+d_{1}-b_{1}\right]\left(k_{1}-M+r_{x}\right) ; \\
M-r_{x} \leq k_{1} \leq M-u_{x} ;
\end{array}\right. \\
& \left\{\begin{array}{l}
J_{2}\left(k_{1}\right)=0,5\left\{\left[\left(M-k_{1}+u_{x}\right)\left(a_{2}-a_{1}\right)+2\left(b_{2}-b_{1}\right)\right]\left(k_{1}-M+u_{x}\right)+\left[u_{x}\left(a_{2}-a_{1}\right)+b_{2}-b_{1}\right]\left(r_{x}-u_{x}\right)\right\} ; \\
M-u_{x} \leq k_{1} \leq M-d_{x} ;
\end{array}\right. \\
& \left\{\begin{array}{l}
J_{3}\left(k_{1}\right)=0,5\left\{\left[\left(M-k_{1}\right)\left(a_{2}-c_{2}\right)+b_{2}-d_{2}+d_{x}\left(a_{2}-a_{1}\right)+b_{2}-b_{1}\right]\left(k_{1}-M+d_{x}\right)+\right. \\
\left.+\left[\left(u_{x}+d_{x}\right)\left(a_{2}-a_{1}\right)+2\left(b_{2}-b_{1}\right)\right]\left(u_{x}-d_{x}\right)+\left[u_{x}\left(a_{2}-a_{1}\right)+\left(b_{2}-b_{1}\right)\right]\left(r_{x}-u_{x}\right)\right\} ; \\
J_{1}\left(k_{2}\right)=0,5\left(\frac{N-d_{x} \leq k_{1} \leq M-l_{x} ;}{c_{1}}-\frac{N-b_{2}-k_{2}}{a_{2}}\right)\left(k_{2}-N+u_{y}\right) ; \\
N-u_{y} \leq k_{2} \leq N-l_{y} ;
\end{array}\right.
\end{aligned}
$$




$$
\begin{gathered}
\left\{\begin{array}{c}
J_{2}\left(k_{2}\right)=0,5\left[\left(\frac{N-2 d_{1}-k_{2}+l_{y}}{c_{1}}-\frac{N-d_{2}-k_{2}}{c_{2}}-l_{x}\right)\left(k_{2}-N+l_{y}\right)+\left(\frac{l_{y}-d_{1}}{c_{1}}-l_{x}\right)\left(u_{y}-l_{y}\right)\right] \\
N-l_{y} \leq k_{2} \leq N-r_{y}
\end{array}\right. \\
\left\{\begin{array}{l}
J_{3}\left(k_{2}\right)=0,5\left\{\left[\left(\frac{N-b_{1}-k_{2}}{a_{1}}\right)-\left(\frac{N-2 d_{2}-k_{2}+r_{y}}{c_{2}}\right)+r_{x}\right]\left(k_{2}-N+r_{y}\right)+\right. \\
\left.+\left[r_{x}-l_{x}+\left(\frac{l_{y}-d_{1}}{c_{1}}\right)-\left(\frac{r_{y}-d_{2}}{c_{2}}\right)\right]\left(l_{y}-r_{y}\right)+\left[\left(\frac{l_{y}-d_{1}}{c_{1}}\right)-l_{x}\right]\left(u_{y}-l_{y}\right)\right\} \\
N-r_{y} \leq k_{1} \leq N-d_{y} .
\end{array}\right.
\end{gathered}
$$

Выражения для случая 3 , когда «перемещение» объекта происходит через линию $y=-N$, находятся аналогично.

Отметим, что при любом направлении «перемещения» объект всегда покидает поле изображения, начиная с края, наиболее удаленного от центра изображения. При этом в выражениях (6)-(11) изменяются диапазоны аргументов $k_{1}$ и $k_{2}$.

Зависимость (6)-(11) от координат точек $A, B, C, D$ дает возможность оценки расположения объекта относительно краев изображения по функции автокорреляции.

\section{Движение объекта}

При обработке видеопоследовательности необходимо не только определить положение объекта на изображении, но и оценить параметры его движения.

Пусть в следующем кадре объект в пределах изображения изменит свое положение на величину $\Delta_{1}$ по горизонтали и $\Delta_{2}$ по вертикали.

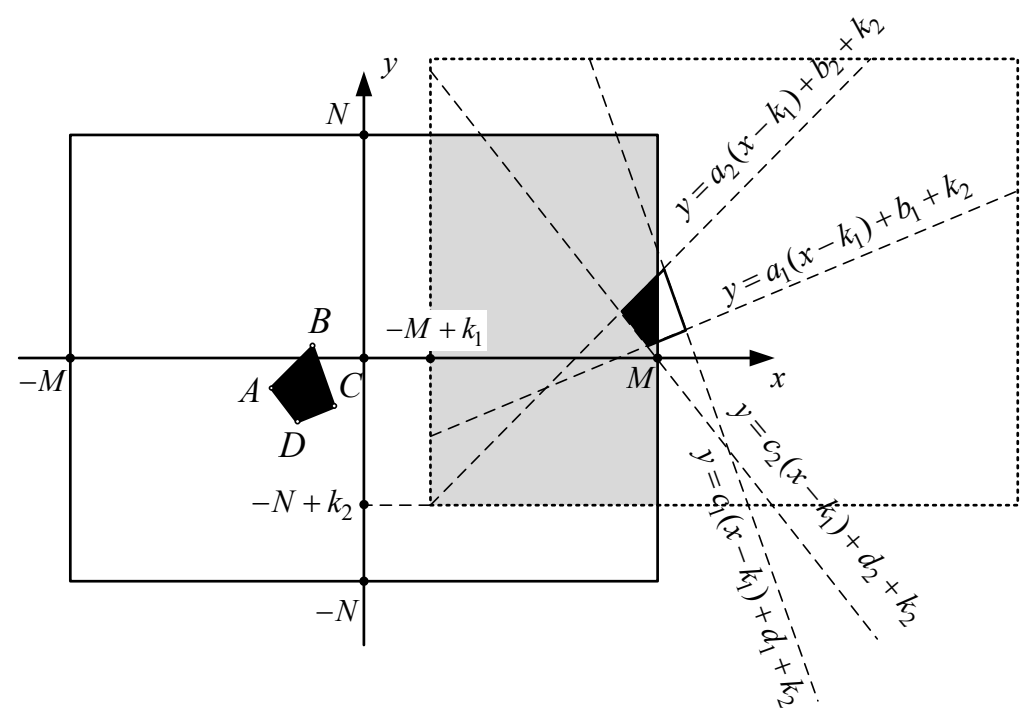

Рис. 4. Схема «перемещения» объекта только через линию $x=M$

Fig. 4. Scheme of "moving" an object only through a line $x=M$ 

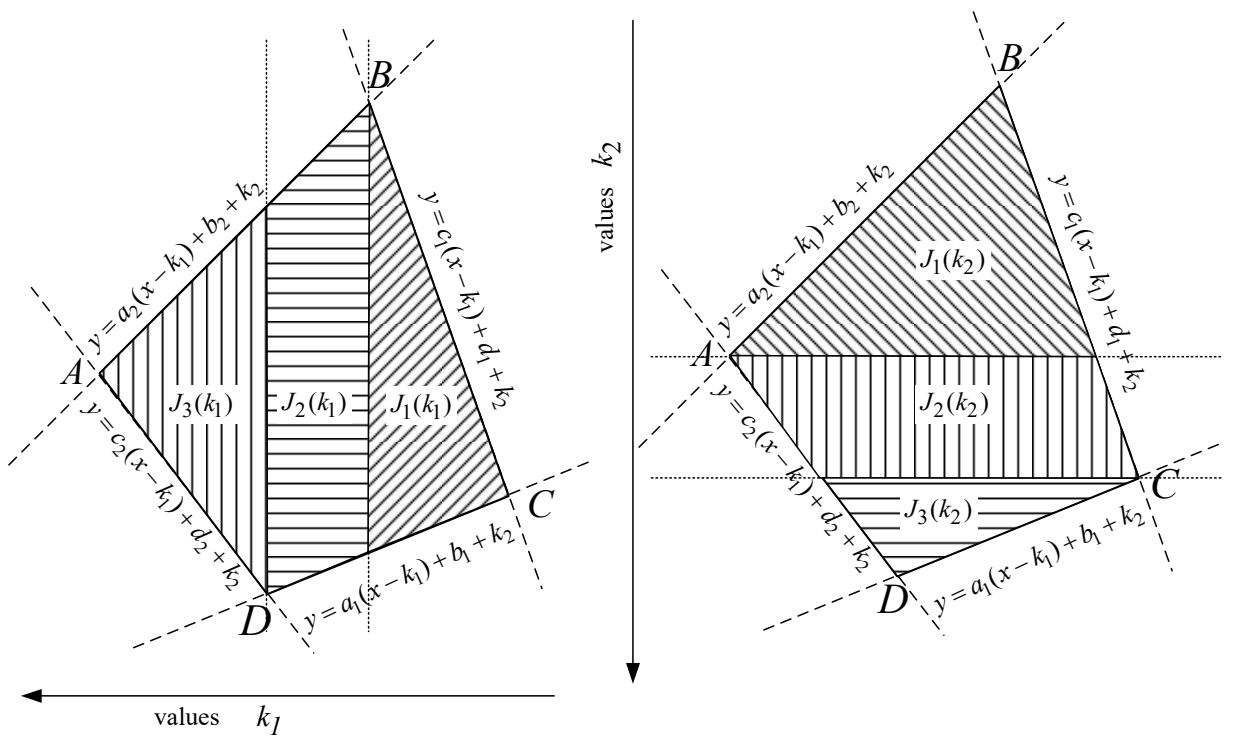

Рис. 5. Изменение площади объекта при «перемещении» через линии $x=M$ и $y=N$

Fig. 5. Change the area of the object when moving through lines $x=M$ and $y=N$

В этом случае для нахождения автокорреляционной функции второго кадра следует в (1)-(2) провести замену: $x \rightarrow x-\Delta_{1}$ и $y \rightarrow y-\Delta_{2}$.

Для идентификации движения найдем разность $\Delta J\left(k_{1}, k_{2}\right)$ функций автокорреляции двух кадров, определяя пределы интегрирования так же, как и в (1)-(2):

$$
\begin{gathered}
\Delta J\left(k_{1}, k_{2}\right)=\iint\left\{\alpha+(\beta-\alpha)\left[\mathbf{1}\left(y-a_{1} x-b_{1}\right)-\mathbf{1}\left(y-a_{2} x-b_{2}\right)\right] \times\left[\mathbf{1}\left(y-c_{2} x-d_{2}\right)-\right.\right. \\
\left.\left.-\mathbf{1}\left(y-c_{1} x-d_{1}\right)\right]\right\} \times\left\{\alpha+(\beta-\alpha)\left[\mathbf{1}\left(y-k_{2}-a_{1} x-b_{1}+a_{1} k_{1}\right)-\mathbf{1}\left(y-k_{2}-a_{2} x-b_{2}+a_{2} k_{1}\right)\right] \times\right. \\
\left.\times\left[\mathbf{1}\left(y-k_{2}-c_{2} x+c_{2} k_{1}-d_{2}\right)-\mathbf{1}\left(y-k_{2}-c_{1} x+c_{1} k_{1}-d_{1}\right)\right]\right\} d x d y- \\
-\iint\left\{\alpha+(\beta-\alpha)\left[\mathbf{1}\left(y-\Delta_{2}-a_{1}\left(x-\Delta_{1}\right)-b_{1}\right)-\mathbf{1}\left(y-\Delta_{2}-a_{2}\left(x-\Delta_{1}\right)-b_{2}\right)\right] \times\right. \\
\left.\times\left[\mathbf{1}\left(y-\Delta_{2}-c_{2}\left(x-\Delta_{1}\right)-d_{2}\right)-\mathbf{1}\left(y-\Delta_{2}-c_{1}\left(x-\Delta_{1}\right)-d_{1}\right)\right]\right\} \times\{\alpha+(\beta-\alpha) \times \\
\times\left[\mathbf{1}\left(y-\Delta_{2}-k_{2}-a_{1}\left(x-\Delta_{1}\right)-b_{1}+a_{1} k_{1}\right)-\mathbf{1}\left(y-\Delta_{2}-k_{2}-a_{2}\left(x-\Delta_{1}\right)-b_{2}+a_{2} k_{1}\right)\right] \times \\
\left.\times\left[\mathbf{1}\left(y-\Delta_{2}-k_{2}-c_{2}\left(x-\Delta_{1}\right)+c_{2} k_{1}-d_{2}\right)-\mathbf{1}\left(y-\Delta_{2}-k_{2}-c_{1}\left(x-\Delta_{1}\right)+c_{1} k_{1}-d_{1}\right)\right]\right\} d x d y .
\end{gathered}
$$

При расчете функции автокорреляции через изменение площади объекта для второго кадра можно воспользоваться выражениями (6)-(11) с заменами $k_{1} \rightarrow k_{1}+\Delta_{1}$ и $k_{2} \rightarrow k_{2}+\Delta_{2}$ соответственно.

В дискретном случае используются отсчеты коэффициента автокорреляции или энергетические характеристики, полученные в [5]:

$$
\sigma\left(k_{1}, k_{2}\right)=\sum_{i=-M}^{M-k_{1}} \sum_{j=-N}^{N-k_{2}} f_{i, j} f_{i+k_{1}, j+k_{2}} \quad \text { при } k_{1}, k_{2} \geq 0 \text {; }
$$




$$
\sigma\left(k_{1}, k_{2}\right)=\sum_{i=-M}^{M-k_{1}} \sum_{j=-N-k_{2}}^{N} f_{i, j} f_{i+k_{1}, j+k_{2}} \quad \text { при } k_{1}, k_{2}<0,
$$

где $f_{i, j}$ - отсчеты видеосигнала изображения.

Сравним результаты для непрерывных и дискретных моделей.

Для моделирования и расчета функции автокорреляции на ЭВМ приняты следующие исходные данные:

- размер изображения $(2 M+1) \times(2 N+1), N=75, M=100$;

- координаты вершин объекта в первом кадре: $A\{-50 ;-35\}, B\{-35 ;-23\}, C\{-28 ;-40\}$, $D\{-38 ;-45\}$.

- «смещение» объекта во втором кадре по горизонтали $\Delta_{1}=17$, по вертикали $\Delta_{2}=29$.

На рис. 6 представлены два кадра изображения, сформированные в соответствии с указанными данными.

На рис. 7 показана разность $\Delta \sigma\left(k_{1}, k_{2}\right)$ энергетических характеристик двух изображений, приведенных на рис. 6.

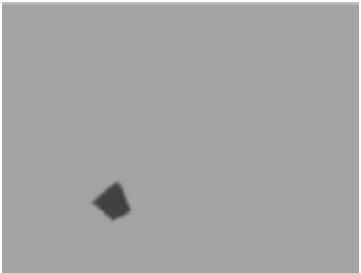

frame 1

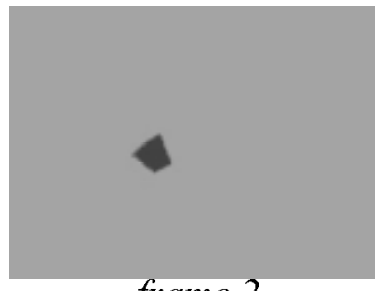

frame 2

Рис. 6. Два изображения с объектом на равномерном фоне

Fig. 6. Two images with an object on a uniform background

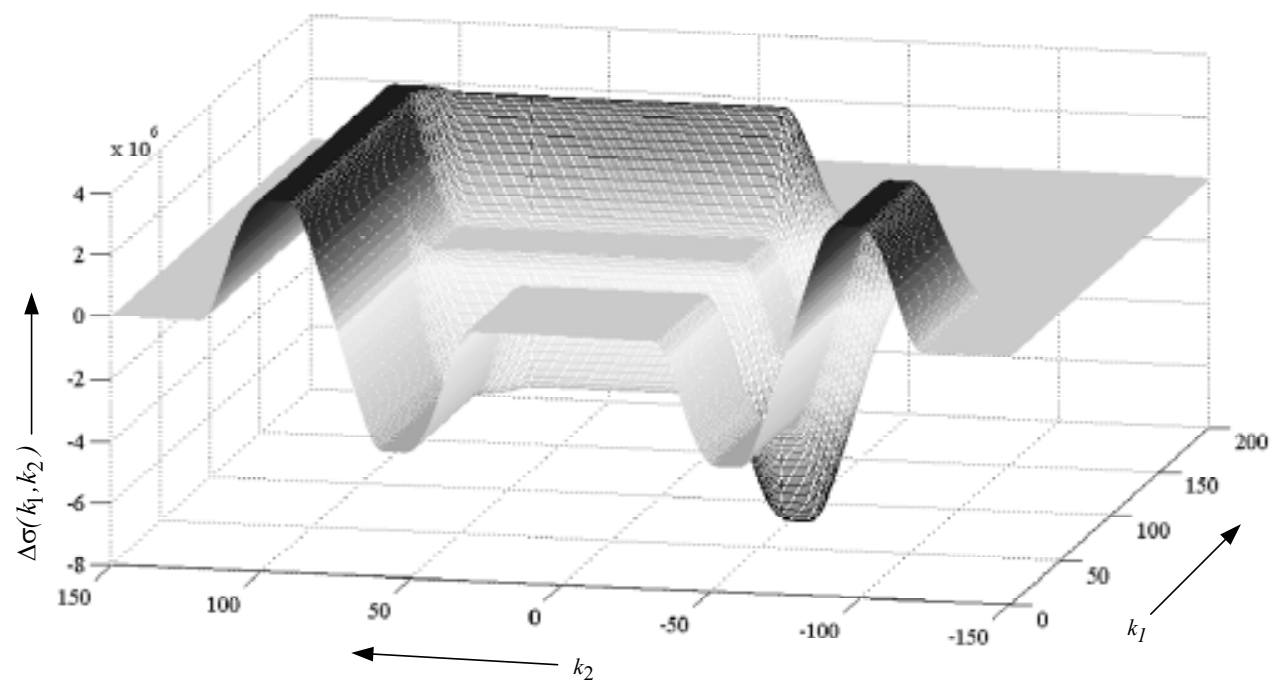

Рис. 7. Разность двумерных энергетических характеристик (дискретный случай)

Fig. 7. The difference between two-dimensional energy characteristics (discrete case) 
Разность $\Delta J\left(k_{1}, k_{2}\right)$ функций автокорреляции, найденных для каждого из двух кадров по выражениям (12), демонстрируется на рис. 8 .

Результат сравнения дискретной и непрерывной модели представлен на рис. 9 в виде разности $\Delta \sigma\left(k_{1}, k_{2}\right)-\Delta J\left(k_{1}, k_{2}\right)$.

Анализ рис. 9 позволяет сделать вывод о совпадении результатов, полученных путем аналитического расчета корреляционных функций через изменение площади объекта, и дискретного случая, расчетные значения для которого выполнены по реальному изображению с помощью ЭВМ. Наличие отклонений большой величины на рис. 9 поясняется тем, что из расчетов были исключены значения корреляционных интегралов для случая «перемещения» объекта изображения через углы изображения. Небольшие отклонения объясняются наличием «переходных» пикселей в реальном изображении. Таким образом, для определения местоположения

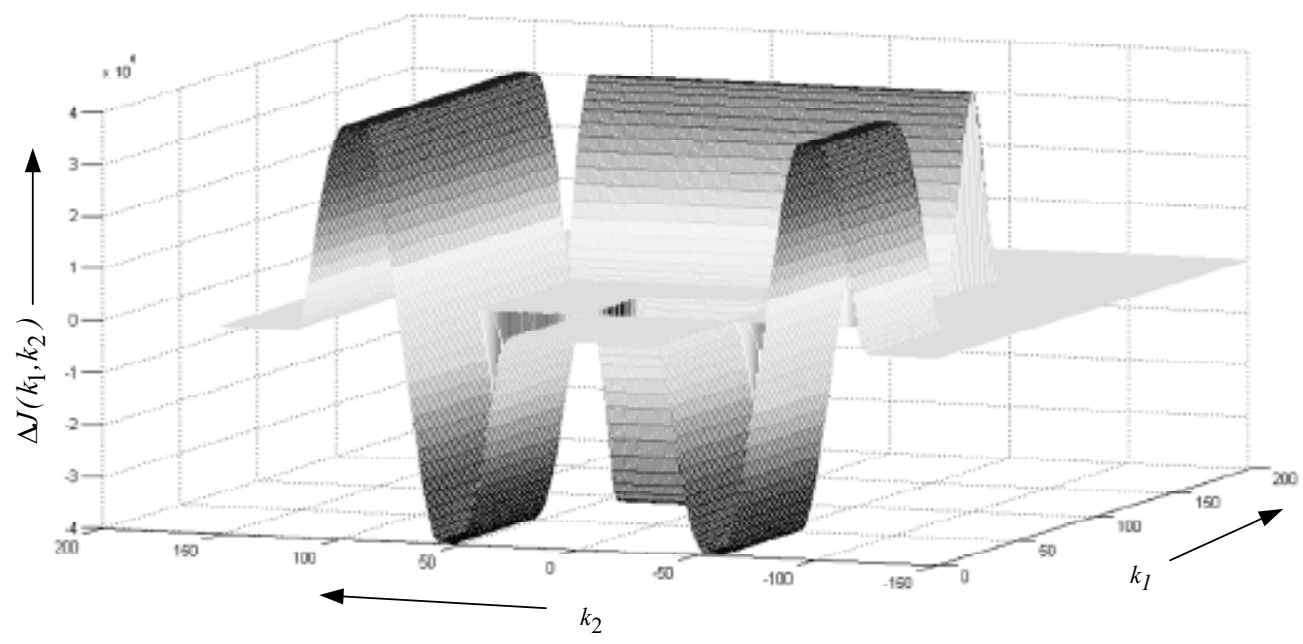

Рис. 8. Разность функций автокорреляции двух кадров (непрерывный случай)

Fig. 8. The difference between autocorrelation functions of two frames (continuous case)

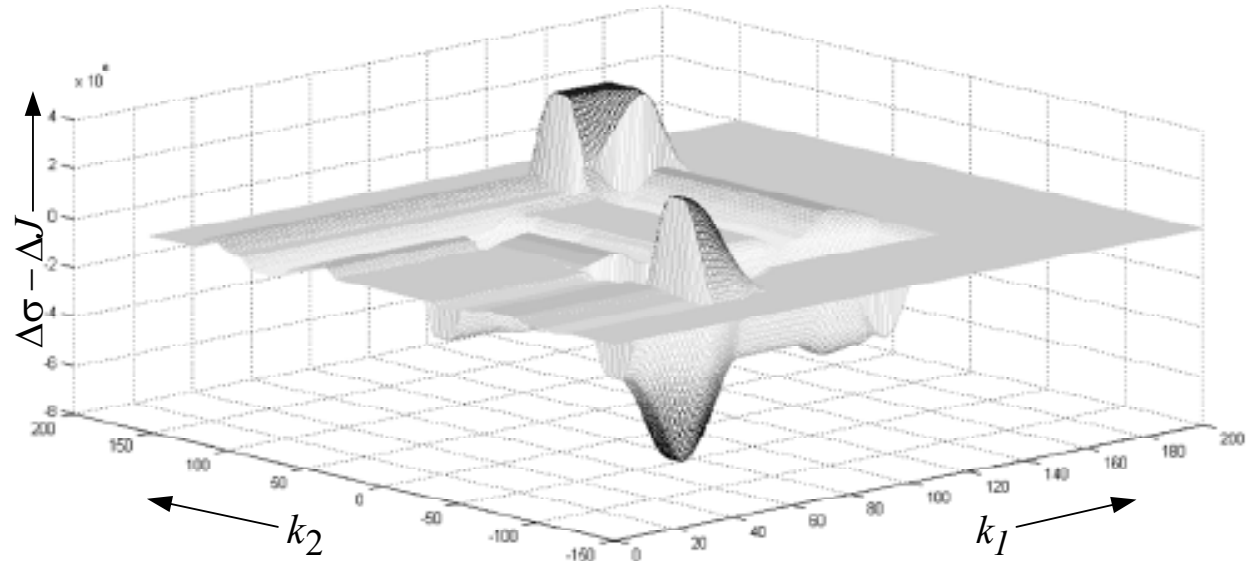

Рис. 9. Сопоставление результатов для дискретной и непрерывной моделей

Fig. 9. Comparison of results for discrete and continuous models 


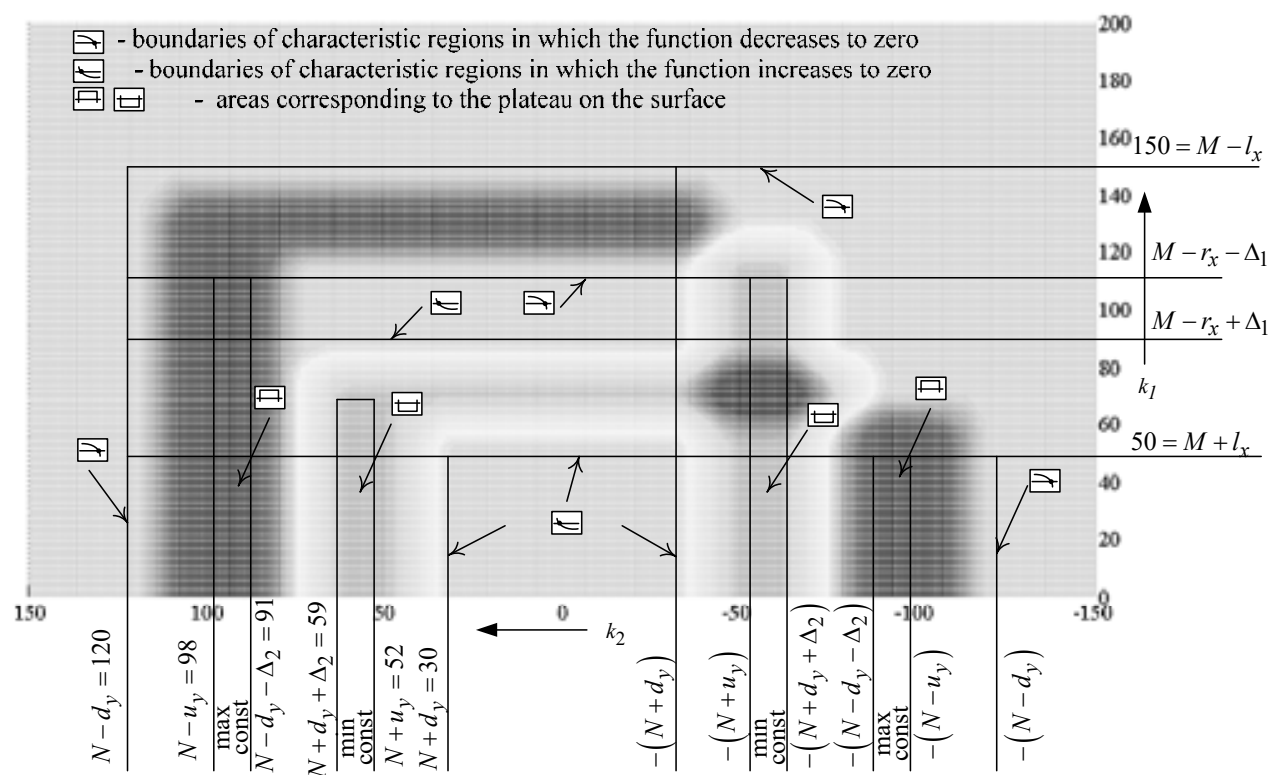

Рис. 10. Линии уровня разности энергетических характеристик двух кадров на плоскости $\left(k_{1}, k_{2}\right)$

Fig. 10. Lines of the level of the difference in the energy characteristics of two frames in the plane $\left(k_{1}, k_{2}\right)$

объекта и параметров его движения можно воспользоваться расчетами, полученными в соответствии с (13)-(14).

Линии уровня функции $\Delta \sigma\left(k_{1}, k_{2}\right)$ образуют характерные области, определяемые параметрами движения объекта и его расположением относительно краев изображения (рис. 10), которые меняют свое положение при различных $\Delta_{1}, \Delta_{2}$. Это позволяет идентифицировать движущиеся объекты по разности их автокорреляционных функций.

Реальные объекты могут не иметь четко выраженных углов, а иметь плавные контуры. Протестируем предлагаемый метод идентификации движущихся объектов при помощи разности автокорреляционных функций на устойчивость к форме объекта. Рассмотрим объект в виде круга постоянной яркости, вписанного в контур четырехугольника $A B C D$ (рис. 11).

При наличии тех же условий межкадрового смещения объекта по горизонтали $\Delta_{1}$, по вертикали $\Delta_{2}$, найдем разность энергетических характеристик $\Delta \sigma \bullet\left(k_{1}, k_{2}\right)$ двух изображений, приведенных на рис. 11. На рис. 12 представлены линии уровня разности энергетических характеристик $\Delta \sigma \cdot\left(k_{1}, k_{2}\right)$.

При сопоставлении рис. 10 и 12 видно, что характерные области остались прежними, однако имеет место незначительное смещение границ на величины $l_{x}^{\prime}, r_{x}^{\prime}, u_{x}^{\prime}, d_{x}^{\prime}$ вследствие изменения удаленности крайних точек объекта относительно центра поля изображения (рис. 11). Это позволяет говорить об устойчивости рассмотренного метода к изменению формы объекта.

Таким образом, можно сделать следующие выводы:

1. Сопоставление двумерных функций автокорреляции, найденных для непрерывного случая через изменение площади объекта, и энергетических характеристик реального изображения говорит о совпадении результатов для непрерывных и дискретных моделей. 

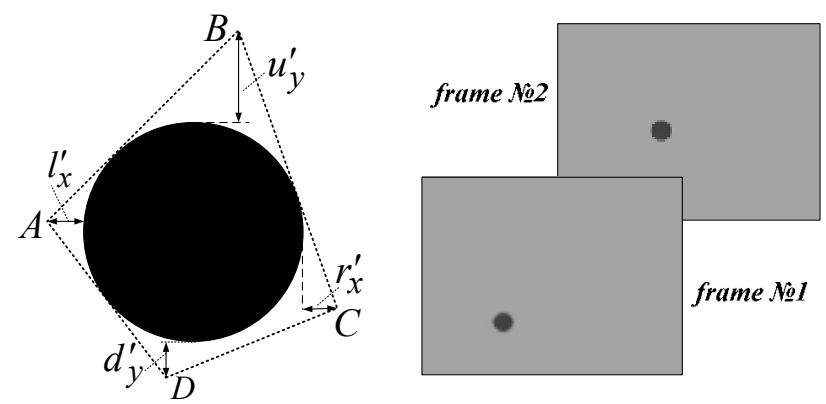

Рис. 11. Круг, вписанный в четырехугольник, и тестовые изображения, содержащие объект такой формы, смещающийся между кадрами

Fig. 11. A circle inscribed in a quadrangle, and test images containing an object of this form, shifting between frames

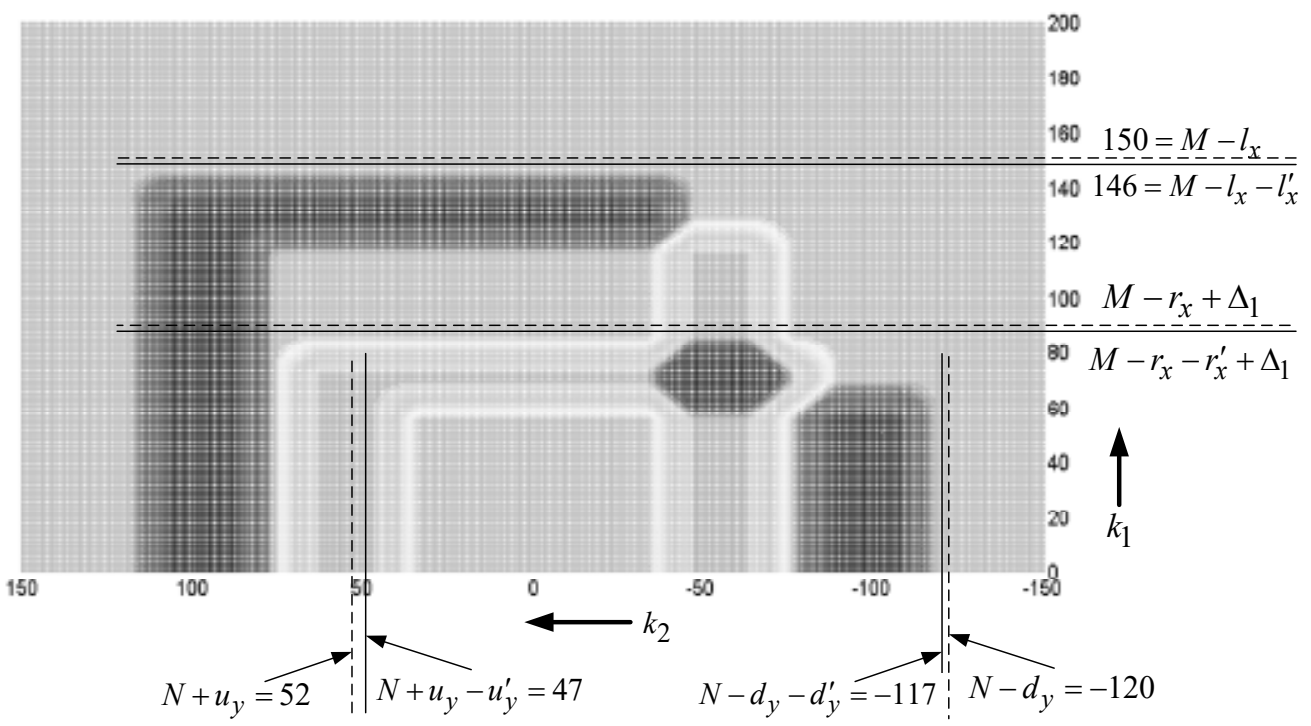

Рис. 12. Результат сравнения разности энергетических характеристик для объектов различной формы

Fig. 12. The result of comparing the difference in energy characteristics for objects of different shapes

2. Предложенный метод позволяет определить положение объекта на изображении, а также найти параметры движения объекта по анализу видеопоследовательности. Рассмотренный метод обладает устойчивостью к изменению формы объекта.

\section{Список литература}

[1] Bogoslovsky A.V., Zhigulina I.V., Maslov I.S., Mordovina T.V. Frequency Characteristics for Video Sequences Processing Smart Innovation, Systems and Technologies. Intelligent Interactive Multimedia Systems and Services. Springer, 2015, 40, 149 - 160.

[2] Богословский А.В., Жигулина И.В., Маслов И.С. Методы обработки видеопоследовательностей на основе анализа энергетических параметров видеосигнала изображения. Радиотехника, 2015, 4, 112 - 119. [Bogoslovsky A.V., Zhigulina I.V., Maslov I.S. Methods of processing 
sequences on the basis of the analysis of the energy parameters of the video image. Radiotehnika, 2015, 4, 112 - 119 (in Russian)].

[3] Bogoslovsky A.V., Zhigulina I.V. A Way of Energy Analysis for Image and Video Sequence Processing. Intelligent Systems Reference Library. Computer Vision in Control Systems-1. Mathematical Theory. Springer, 2015, 73, $183-210$.

[4] Богословский А.В., Сухарев В.А., Жигулина И.В., Пономарев А.В. Корреляционный анализ финитных по пространству одномерных сигналов. Радиотехника, 2017, 12, 4 - 7. [Bogoslovsky A.V., Sukharev V.A., Zhigulina I.V., Ponomarev A.V. Models of the energy characteristics of the video image. Radiotehnika, 2017, 12, 4 - 7 (in Russian)].

[5] Богословский А.В., Жигулина И.В. Методология построения моделей межкадровых разностей частотных характеристик. Радиотехника, 2013, 9, 76 - 82. [Bogoslovsky A.V., Zhigulina I.V. Methodology of construction of models of the frame difference of frequency characteristics. Radiotehnika, 2013, 9, 76 - 82 (in Russian)]. 